\title{
Surface Modification of Membranes for Fouling Reduction
}

\author{
Jochen Meier-Haack ${ }^{1}$, Kornelia Schlenstedt, Christian Langner \\ Leibniz Institute of Polymer Research Dresden, Hohe Straße 6, 01069 Dresden, Germany
}

\begin{abstract}
Despite great effort that has been made to reduce and understand fouling, this phenomenon is still a major problem in membrane applications. Numerous methods, both from a chemical and engineering point of view, have been introduced to overcome this problem. In this contribution, we report on the modification of membranes with polyelectrolytes and polyelectrolyte multilayers utilizing two of the mentioned strategies. The effect of surface modification on the fouling behavior as well as on the critical flux will be discussed on two examples, microfiltration membranes and RO membranes.
\end{abstract}

Keywords: Fouling, surface modification, polyelectrolytes, grafting, critical flux

\section{Introduction}

Despite great effort that has been made to reduce and understand fouling, this phenomenon is still a major problem in membrane applications. Several factors such as hydrophobicity, surface charge and operating mode are effecting the deposition of feed components at the membrane surface and thus causing fouling. In the past, numerous methods based on both chemical and engineering methods, have been introduced to overcome this problem [1-10]. In order to illustrate the fouling problem, SEM images of polypropylene hollow fiber membranes before (a) and after (b) protein filtration are shown in Figure 1.
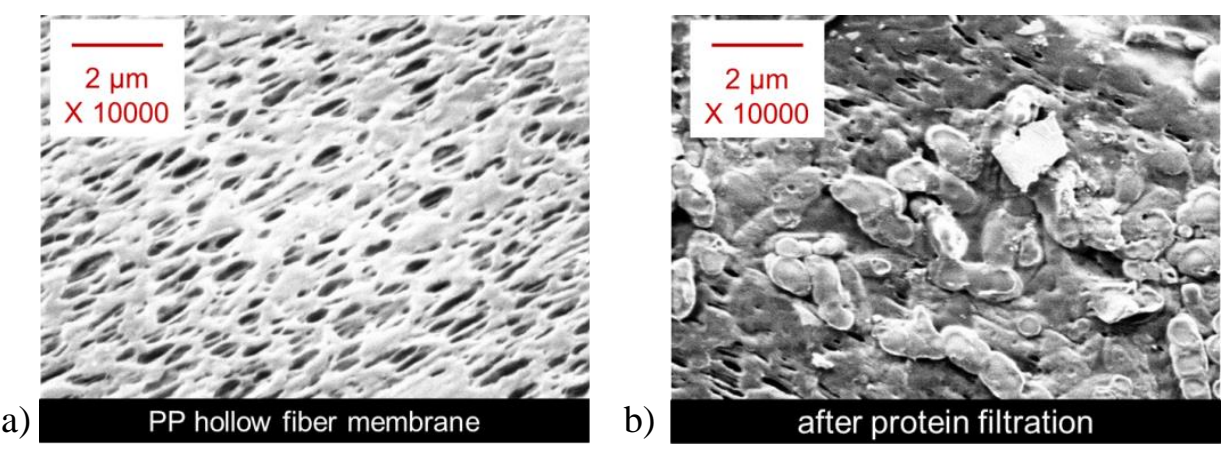

Figure 1: SEM images of a PP hollow fiber a) before and $b$ ) after protein filtration

Figure 2 outlines different strategies for the surface modification of membranes to alter the surface properties. In this contribution, we report on the modification of membranes with polyelectrolytes and polyelectrolyte multilayers utilizing two of the mentioned strategies. The

${ }^{1}$ Corresponding author email: mhaack@ipfdd.de 
effect of surface modification on the fouling behavior as well as on the critical flux will be discussed on two examples, microfiltration membranes and RO membranes.

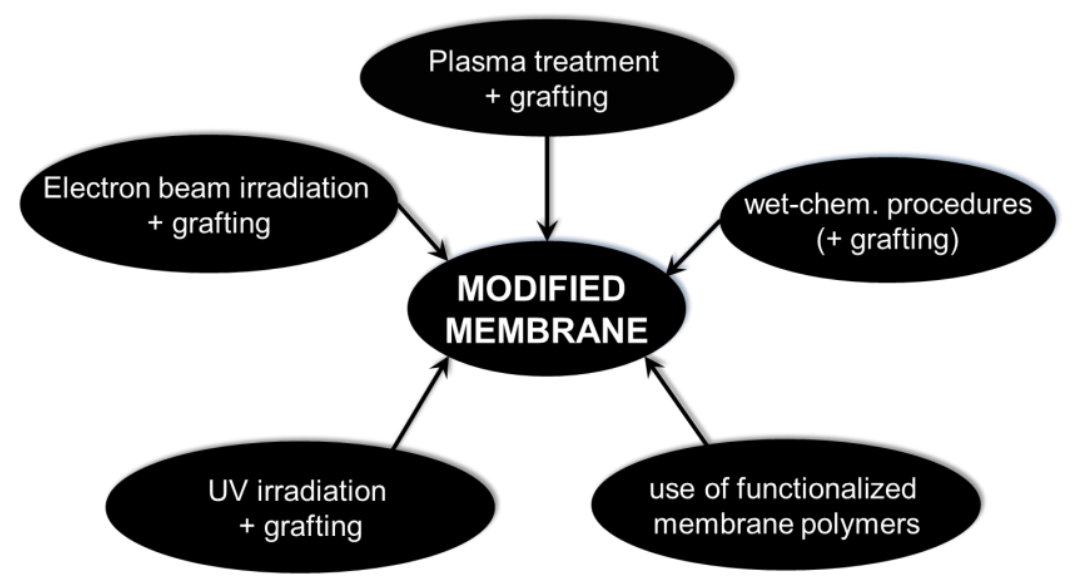

Figure 2: Strategies for surface modification of membranes

\section{Surface modification of microfiltration membranes}

Surface modification of polypropylene (PP) microfiltration membranes was initiated by either plasma treatment or coating with a macro-initiator (perester containing maleic acid copolymer) and subsequent grafting of polyacrylic acid (PAAc) [8, 11, 12]. The effect of different treatment steps (plasma treatment, grafting, and polyelectrolyte layer build-up) on the surface and filtration properties are shown in Figure 3. The unmodified PP membrane shows the typical $\mathrm{pH}$ dependence of the $\zeta$-potential (Figure $3 a$ ) of a hydrophobic surface with no functional groups $(\bigcirc)$. The negative surface charge is explained by the preferred adsorption of anions. On plasma treatment and grafting of the first PAAc layer, the isoelectric point (IEP; net charge $=0$ ) shifts to a lower $\mathrm{pH}$ value $(\mathrm{pH} 3.5)$ and the surface charge is negative over a wide $\mathrm{pH}$ range $(\square, \nabla)$. The formation of a plateau at higher $\mathrm{pH}$ values indicates the presence of carboxylic acid groups at the surface. On adsorption of a polycation (polydiallyldimethyl ammonium chloride (PDADMAC); $\triangle$ ) the IEP shifts to higher $\mathrm{pH}(\mathrm{pH}$ 8 ) and the surface charge is positive over a wide $\mathrm{pH}$ range. Further adsorption of PAAC leads to a reversal of the surface charge $(\diamond)$. Thus, the polyelectrolyte multilayer (PEL) build-up allows for the adjustment of the surface charge and for the control of electrostatic interactions between feed components and the membrane surface.

The different treatment steps also affect the filtration properties. The plasma treatment leads $(\square)$ to an increase of permeate flux due to hydrophilization of the surface and partial destruction of the pore structure (pore opening). This effect is compensated by grafting of a first PAAc layer $(\nabla)$. Now the grafted polymer chains cover the pores resulting in a pore blockage. Interestingly, adsorption of a layer pair consisting of a polycation (PDADMAC) and a polyanion (PAAc) does not cause a further decrease in flux. On the contrary the flux increased to the same level as observed for the unmodified membrane $(\triangle)$, which can be explained by compaction of the grafted/adsorbed layers because of the polyelectrolyte complex formation. The adsorption of a second layer pair resulted in a higher pore blockage 
and thus in a lower permeate flux $(\diamond)$. However, the flux of this 5-layer membrane is in the same range as the flux of the single layer membrane $(\nabla)$.

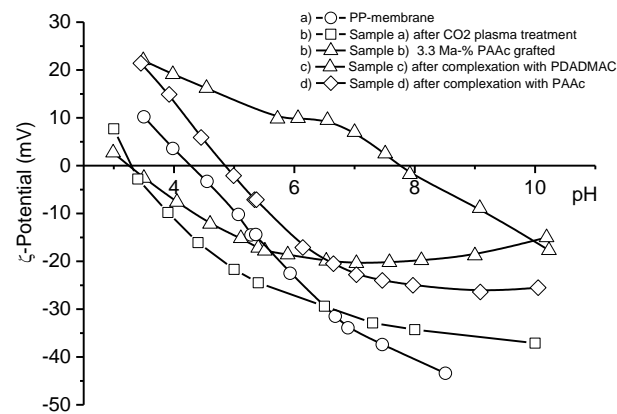

a)

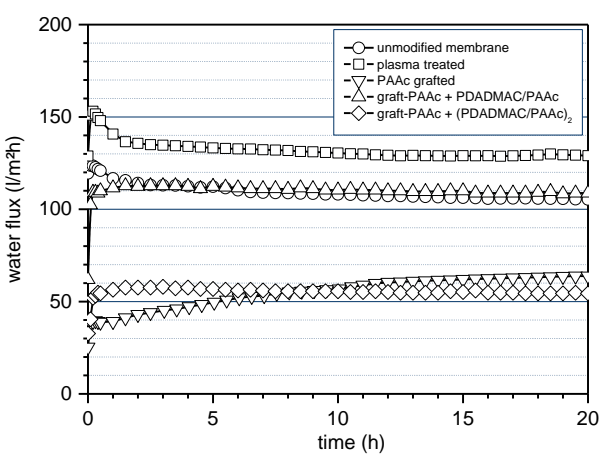

b)

Figure 3: Effect of surface modification on (a) surface charge and (b) filtration properties of PP membranes (transmembrane pressure 3 bar)

The "critical flux" $\mathrm{J}_{\text {crit. }}$ is defined as the permeate flux below which no irreversible fouling occurs [13-15]. It is determined by flux-stepping experiments [16] and the mean value of flux of each transmembrane pressure (TMP) step is plotted over TMP. In the case of no fouling, a linear relationship between flux and TMP is obtained (Figure 4). As soon as fouling occurs, this relationship deviates from linearity. As a result, graft modification of PP-MF-membranes with PAAc results in an enhancement of the critical flux. However, due to the pore size restriction by the graft layer a much higher transmembrane pressure was needed to maintain the same permeate flux as for the unmodified membranes. Since the chain conformation of the grafted polyelectrolyte layers in polyelectrolyte complexes is more compact than in single layers larger pores can be expected. Thus, a much higher flux at similar transmembrane pressures compared to the single layer modified membranes was observed. Consequently, the PEL-modified membranes showed higher permeate flux at lower pressure as compared to the single layer modified membranes and a higher critical flux. However, in both cases the weak form of critical flux $\left(\mathrm{J}_{\text {water }}>\mathrm{J}_{\text {protein }}\right)$ was observed. The situation changed when filtrating an aqueous BSA solution at $\mathrm{pH} 7.9$ through the unmodified membrane. At low fluxes/TMPs (up to $20 \mathrm{~L} / \mathrm{m} 2 \mathrm{~h}$ and 0.3 bars), the permeability was approximately the same as for the sodium bicarbonate solution. With increasing flux/TMP, the data points deviate from those of the pure solvent filtration experiment. For a given flux, a higher TMP was needed than in the solvent filtration experiment, indicating the start of the fouling process. From these results, which are visualized in Figure 4, a strong form of fouling during the filtration of a BSA solution through an unmodified PP-membrane could be deduced. It should be noted, that the protein retention was in the range from $93 \%$ to $98 \%$ in the experiments conducted at fluxes up to $40 \mathrm{~L} / \mathrm{m}^{2} \mathrm{~h}$. Only at a flux of $80 \mathrm{~L} / \mathrm{m}^{2} \mathrm{~h}$, the retention was lowered to $60 \%$. 


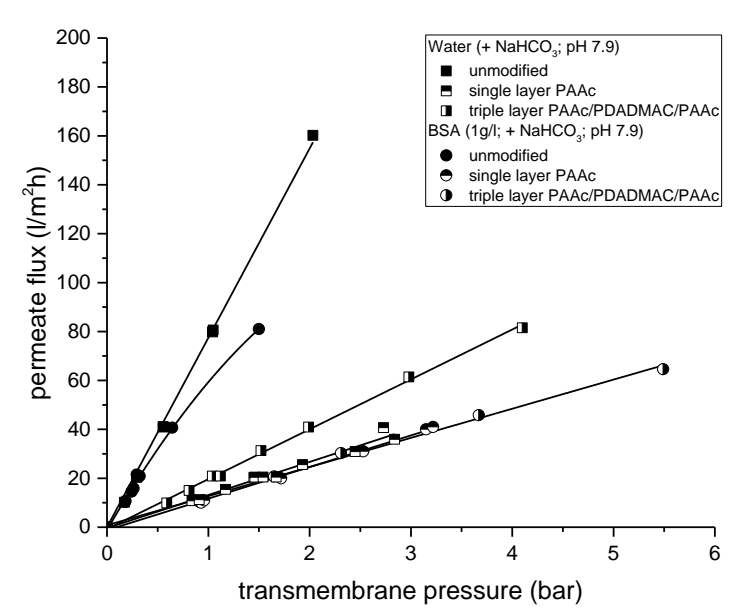

Figure 4: Flux-pressure relationship for sodium bicarbonate solution ( $p H$ 7.9) and a BSA solution at pH 7.9 derived from flux-stepping experiments for unmodified and modified PP-membranes.

\section{Surface modification of thin film composite (TFC) reverse osmosis membranes}

Reverse osmosis or nanofiltration membranes consist of three distinctive layers, namely a polyester (PET) non-woven, a PES layer and an aromatic polyamide (PA) layer. The structure of such thin film composite membranes is shown in Figure 5. The thicknesses of the individual layers are $\sim 100 \mu \mathrm{m}$ for PET layer and the PES layer and $\sim 100 \mathrm{~nm}$ for PA layer. The non-woven and the PES layer act only as support for the PA layer, which is responsible for the separation. The process for preparation of TFC RO membranes is schematically outlined in (Figure 6a). For the modification of reverse osmosis (RO) membranes two different approaches were applied. On one hand carbonyl chloride groups remained at the surface after the formation of the polyamide layer by interfacial polycondensation were used for grafting of amine groups containing hyperbranched polymer (Figure 6b) [17]. In the second approach, monomers containing ethinyl groups were incorporated into the polyamide layer. These groups were reacted with azide-groups containing hydrophilic polymers by copper catalyzed azide alkyne cycloaddition (Figure $6 b$ ).

a)
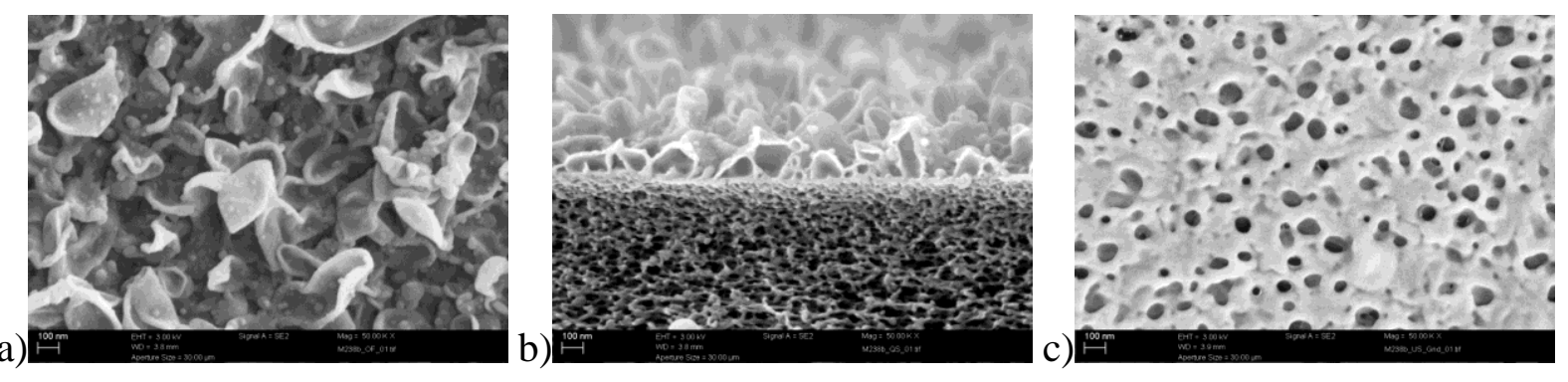

Figure 5: SEM images of a thin film composite membrane: a) surface, b) cross section, c) bottom of PA layer

On modification of RO membranes with a polycation (hyperbranched PAMAM) during the preparation of the active PA layer, the formation of a distinct but highly permeable second layer, mainly consisting of PAMAM was observed. The modification led to a more hydrophilic (lower water contact angle) and less negatively charged surface as compared to the unmodified RO membrane. The grafting of neutral hydrophilic polymers resulted again in 
a more hydrophilic surface, but the surface charge was not influenced compared to the unmodified membrane.

a)

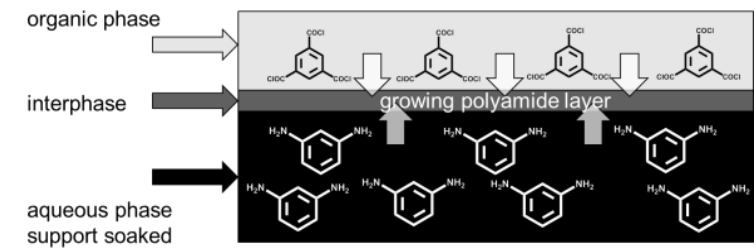
) b)

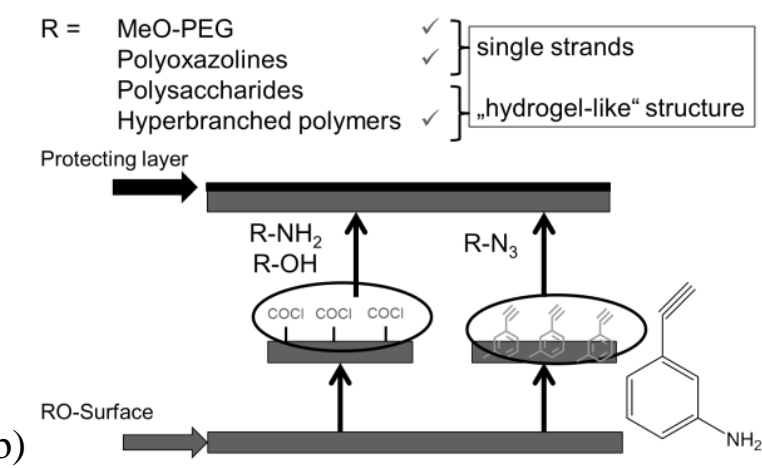

Figure 6: Preparation towards surface modified RO membranes: a) formation of polyamide layer by interfacial polycondensation; $b$ ) surface modification by grafting of hydrophilic polymers

The filtration properties like permeate flux and salt rejection were not influenced by the modification step as outlined in Figure 7a. However, distinct differences with protein (bovine serum albumin; BSA) were detected during filtration experiments Figure $7 b$. For the unmodified membrane, a steep drop in flux $(6 \% / \mathrm{h})$ by during protein filtration was observed (Figure $7 b$ ). After rinsing with clean water, only $90 \%$ of the original flux was reached, showing a relatively high irreversible protein fouling. The modified membranes showed again a flux decline during protein filtration. Yet, this effect was less pronounced as for the unmodified membrane $(\sim 1.5 \% / \mathrm{h})$. The ability to clean the modified membranes with pure water is much better than of the unmodified membrane. However, some differences were observed between the different membranes. While the flux of the PAMAM modified membrane could only be recovered by $90 \%$, the flux recovery of the PEG or PMeOx modified membranes was almost $100 \%$. This result can be attributed to ionic interaction or hydrogen bonding between the amino groups of PAMAM and BSA molecules, while the PEG or PMeOx chains have no or much weaker interactions with BSA.

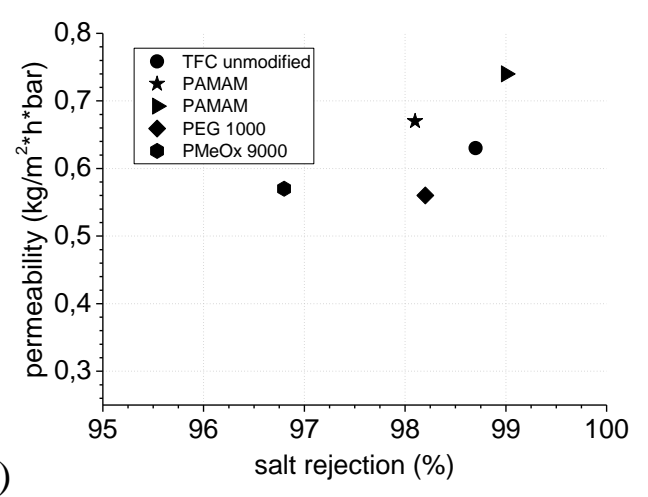

Figure 7: Filtration and fouling properties of TFC RO membranes; effect of modification on a) permeate flux and salt rejection and b) fouling and cleanability

Another problem that occurs in application of polyamide based membranes is the sensitivity towards chlorine, which is frequently used in the form of hypochlorite for disinfection. The free chlorine reacts with the polyamide leading to chlorinated polyamide $(\mathrm{N}-$ 
chlorination as well as C-chlorination (Orthon rearrangement)) and degradation of the PA layer, as can show in Figure $8 a$. The lifetime of RO membranes regarding degradation by chlorine treatment can be significantly improved by surface modification. However, not all polymers used for surface modification are suitable to protect the PA layer from degradation. The PAMAM modified membrane shows the best chlorine stability followed by the PMeOxgrafted membrane. The PEG modification has no effect on chlorine stability (Figure $8 b$ ). Especially the high chlorine stability of the PAMAM modified membrane, which is almost double that of the unmodified membrane, is on one hand attribute to the high coverage of the surface with PAMAM. On the other hand, the amide and amino groups might act as chlorine scavenger and as sacrificial layer, which is degraded first.

a)

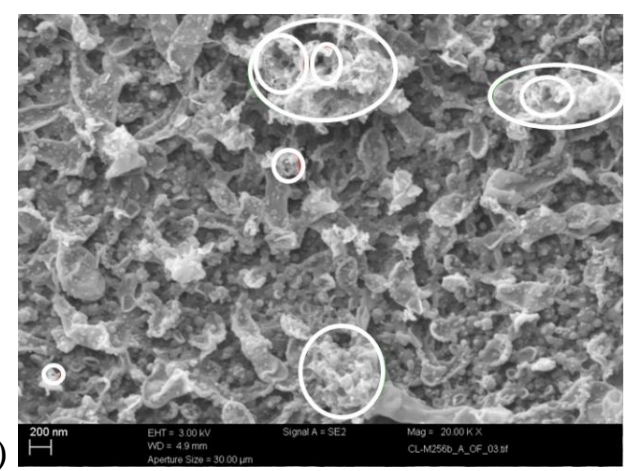

b)

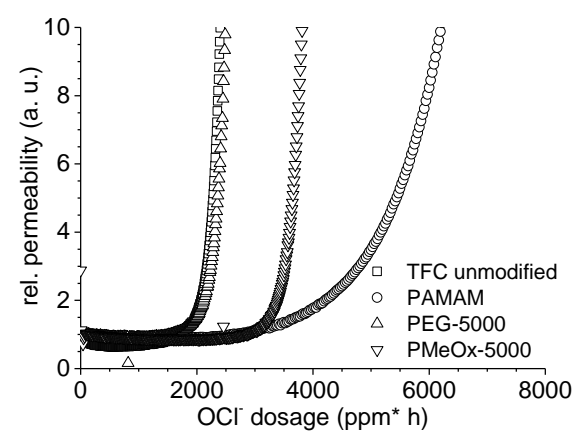

Figure 8: Effect of chlorine treatment on a) the membrane performance (permeate flux) and $b$ ) the morphology of RO membranes (severe corrosion (degradation) are indicated by white circles)

\section{References}

[1] Belfer S., Purinson Y., Fainshtain R., Radchenko Y., Kedem O., Surface modification of commercial composite polyamide reverse osmosis membranes, J. Membr. Sci. 139 (1998) 175-181.

[2] Benhabbour R.S., Sheardown H., Adronov A., Protein Resistance of PEG-Functionalized Dendronized Surfaces: Effect of PEG Molecular Weight and Dendron Generation, Macromolecules 41 (2008) 4817-4826.

[3] Chamoulaud G., Bélanger D., Chemical modification of the surface of a sulfonated membrane by formation of a sulfonamide bond, Langmuir 20 (2004) 4989-4995.

[4] Cho S.A., Cho E.A., Oh I., Kim H., Ha H.Y., Hong S., Ju J.B., Surface modified Nafion membrane by ion beam bombardement for fuel cell applications, J. Power Sources 155 (2006) 286-290.

[5] Johnsen K., Kirkhorn S., Olafsen K., Redford K., Stori A., Modification of Polyolefi Surfaces by Plasma-Inducted Grafting, J. Appl. Polym. Sci. 59 (1996) 1651-1657.

[6] Kim J., Park P., Lee C., Kwon H., Surface modification of nanofiltration membranes to improve the removal of organic micro-pollutants (EDCs and PhACs) in drinking water treatment: Graft polymerization and cross-linking followed by functional group substitution, J. Membr. Sci. 321 (2008) 190-198.

[7] Mougenot P., Koch M., Dupont I., Schneider Y., Marchand-Brynaer J., Surface Functionalization of Polyethylene Teraphthalate Film and Membranes by Controlled Wet Chemistry, J. Coll. Interface Sci. 177 (1996) 162-170.

[8] Rieser T., Lunkwitz K., Berwald S., Meier-Haack J., Müller M., Cassel F., Dioszeghy Z., Simon F., in: I. Pinnau, B.D. Freeman (Eds.) Membrane Formation Modification, American Chemical Society, Washington DC 2000 pp. 189-204, ISBN 0-8412-3604-6. 
[9] Ulbricht M., Advanced functional polymer membranes, Polymer (2006) 2217-2262.

[10] Berwald, S.; Lenk, W.; Rieser, T.; Meier-Haack, J.; Eichhorn, K.-J.; Lunkwitz, K., Modifizierung von Polyaramid-Ultrafiltratonsmembranen durch Elektronenbestrahlung, Angew. Makromol. Chem. 273 (1999) 47-52.

[11] Carroll T., Booker N. A., Meier-Haack J., Polyelectrolyte-grafted microfiltration membranes to control fouling by natural organic matter in drinking water, J. Membr. Sci. 203 (2002) 3-13.

[12] Meier-Haack J., Derenko S., Seng J., Fouling reduction by graft-modification with hydrophilic polymers, Sep. Sci. Technol. 42 (2007) 2881-2890.

[13] Field R.W., Wu D., Howell J.A., Gupta B.B., Critical flux concept for microfiltration fouling, J. Membr. Sci. 100 (1995) 259-272.

[14] Howell J.A., Sub-critical flux operation of microfiltration, J. Membr. Sci. 107 (1995) 165-171.

[15] Bacchin P., Aimar P., Field R.W., Critical and sustainable fluxes: Theory, experiments and applications, J. Membr. Sci. 281 (2006) 42 - 69.

[16] van der Marel P., Zwijnenburg A., Kemperman A., Wessling M., Temmink H., van der Meer W., An improved flux-step method to determine the critical flux and the critical flux for irreversibility in a membrane bioreactor, J. Membr. Sci. 332 (2009) 24 - 29.

[17] Nikolaeva D., Langner C., Abdel Rehim M., Voit B., Meier-Haack J., Hydrogel surface modification of reverse osmosis membranes, J. Membr. Sci. 476 (2015) 264-276. 